\title{
Blocking Probabilities in WDM Switching Networks using Overflow Analysis Method
}

\author{
Arif Ali Rehman, Abid Karim and Shakeel Ahmed Khoja \\ Bahria University (Karachi Campus) 13 National Stadium Road 75260 Karachi Pakistan \\ \{rehmanaf, abid, shakeel\}@bimcs.edu.pk
}

\begin{abstract}
Wavelength Division Multiplexed switching networks are considered as an important candidate for the future transport networks. As the size of network increases conventional methods used in teletraffic theory to model these networks become computationally difficult to handle as the state space grows exponentially. In this research we have applied overflow analysis to model these networks. Our results show that moment analyses using equivalent random theory (ERT) results in accurate approximations for the modeling of WDM switching networks.
\end{abstract}

Keywords: WDM switching networks, Equivalent Random Theory (ERT)

\section{Introduction}

For communication networks such as wireless or wavelength division multiplexing optical networks with wavelength conversion capabilities, the teletraffic modeling results in a multidimensional systems. Glenstrup. et. al. [1] has demonstrated an exact method for the calculation of the blocking probability of small size systems. Yates. et. al [2] has developed an approximate method to calculate the blocking probabilities using graph-theoretical approach for the networks with fixed routing with limited conversion degree. Subramanium et. al. [3] has focused on conversion density using Bernoulli-Poisson-Pascal (BPP) for the evaluation of blocking probability; however for large systems these methods face limitations due to the exponential growth of state space[4].

In this paper we have shown the use of overflow analysis method for calculating blocking probability of the given network. Our approach is applicable for the WDM switching networks ranging from no conversion to full conversion capability, with any numbers of fibers and wavelengths.

In section 2, a theoretical model is presented to describe the WDM network. Section 3 discusses the analytical approach used for the calculation of blocking probability of the network, whereas discussion of resulted is presented in section 4 . The analytical results are shown to agree well with the network simulation. 


\section{Theoretical Model}

Consider a WDM switching network as shown in Figure 1. There are $N_{l}$ incoming fibers and $\mathrm{N}_{2}$ outgoing fiber links to the network. The multiplexing degree which corresponds to the number of incoming channels (wavelengths) per fiber is $M$. The network has a provision of a pool of " $\mathrm{K}$ " wavelength converters. Each incoming wavelength from the fiber is separated using de-multiplexers.

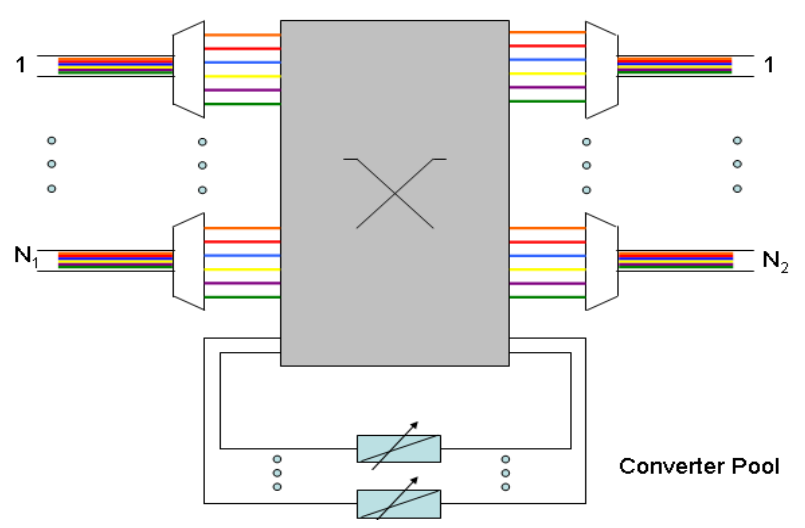

Fig. 1. WDM Network Model

The internal design of the switching node switches the incoming connection requests of particular wavelengths to switching elements specific for those wavelengths. The switched wavelength is sent to the multiplexer of the respective route. In case if the wavelength on the outgoing wavelength is already occupied then the incoming connection request is internally switched to tunable wavelength converters Each tunable wavelength converter can receive wavelength from any switching element and converts to the wavelength which is free on the outgoing fibers and send the wavelength to the respective switching element.

Considering switching network as primary group of $\mathrm{n}_{1}$ servers and wavelength converters as secondary groups $n_{2}$ servers respectively (fig. 2 ), the above system can be assumed as a overflow traffic model with following assumptions.

- The arriving requests exhibit Poisson distribution with intensity $\lambda$.

- The holding time distribution is negative exponential with parameter $\mu$.

- With parameters $\lambda$ and $\mu$ the intensity of offered traffic is given as $\lambda / \mu$.

- The incoming request is initially offered to primary servers.

- If all primary servers are occupied then the traffic is over flown to the secondary servers forming an interrupted Poison process (IPP) with traffic intensity $\alpha$.

- If all the servers in secondary group are occupied then the incoming request is completely blocked.

- The connection on the converted wavelength will simultaneously keep the possession of wavelength converter as well as outgoing fiber as long as it exists.

- The primary servers have not only to serve the incoming requests but also the converted wavelength requests. 
- With each converted wavelength the number of servers is reduced to serve the new incoming requests.

The primary and secondary groups jointly form loss system where number of servers is equal to $n_{1}+n_{2}$. The division of the capacity into two parts does not affect the overall behavior of the system, as it always forms $\mathrm{M} / \mathrm{M} / \mathrm{n}$ system.
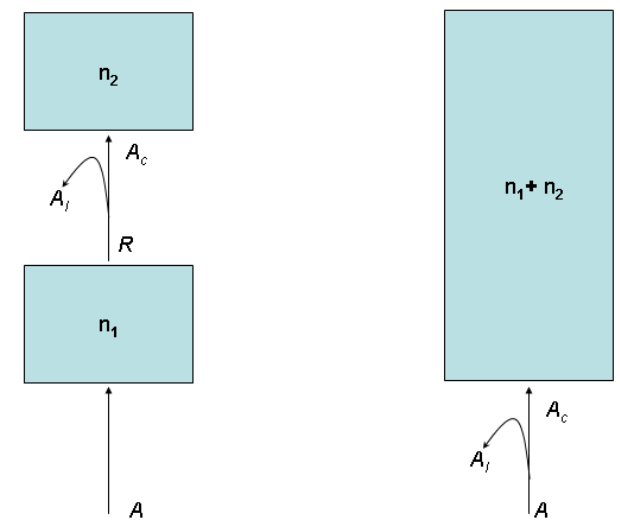

Fig. 2. Block diagram representation of overflow system.

Equivalent random theory (ERT) method provides an approximate method to calculate the blocking probability for non-Poisson traffic. The traffic is defined using mean intensity $\mathrm{R}$ and variance $\mathrm{V}$ of the occupancy in an infinite system. For the case of several independent contributors the mean intensity and variance is equal to the sum of individual mean intensities and variances respectively. The idea of ERT method is to get a traffic (R, V) from a fictitious channel with offered traffic $A^{*}$ and number of servers $\mathrm{N}^{*}$. The values of $\mathrm{A}^{*}$ and $\mathrm{N}^{*}$ are such calculated that the overflow traffic in the fictitious channel has intensity $\mathrm{R}$ and $\mathrm{V}$. The values of $\mathrm{A}^{*}$ and $\mathrm{N}^{*}$ are calculated numerically as described in [5 and 6]. Thereafter the blocking in the overflow channel is calculated as:

$$
\frac{A^{*} E\left(N_{1}+N^{*}, A^{*}\right)}{R} .
$$

And the total blocking of the system is calculated as:

$$
\frac{A^{*} E\left(N_{1}+N^{*}, A^{*}\right)}{\sum_{i} A_{i}}
$$




\section{Results and Discussion}

For calculations of blocking probability five fibers, fifty wavelengths, while varying the number of wavelength converters used ranging from no conversion to full conversion. Fig. 3 shows the result of numerical analyses as performed using algorithms of one moment (solid lines) and two moment (dotted lines) methods respectively described in [7]. The results indicate that one moment analysis underestimates the blocking probability for small value of offered load; however for large traffic loads predictions from both methods i.e. one moment and two moments are coincident. The figure also indicates the significant improvement in the blocking characteristics with the presence of wavelength conversion. It is evident from the graph that the presence of limited number of converters results in blocking probabilities that are closer to full conversion case.

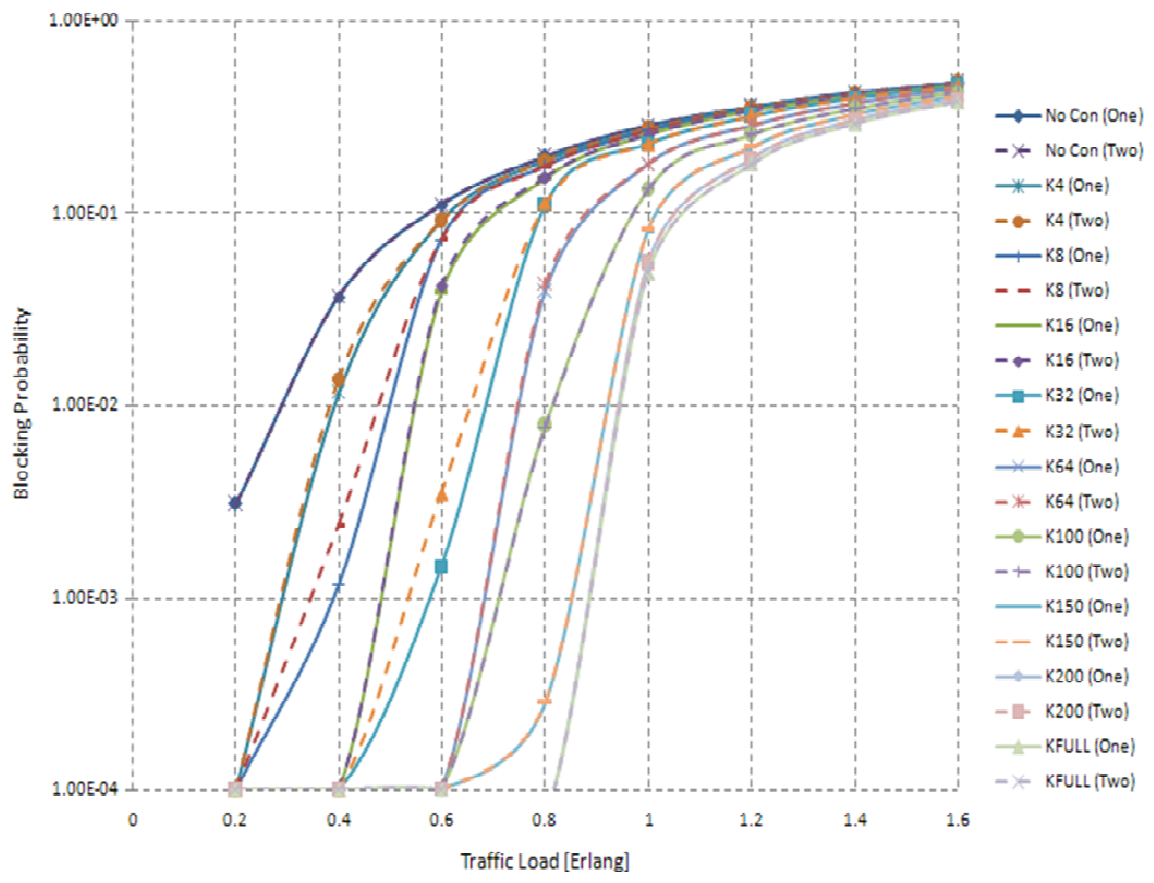

Fig. 3. Results of one moment and two moments analyses [7]. 


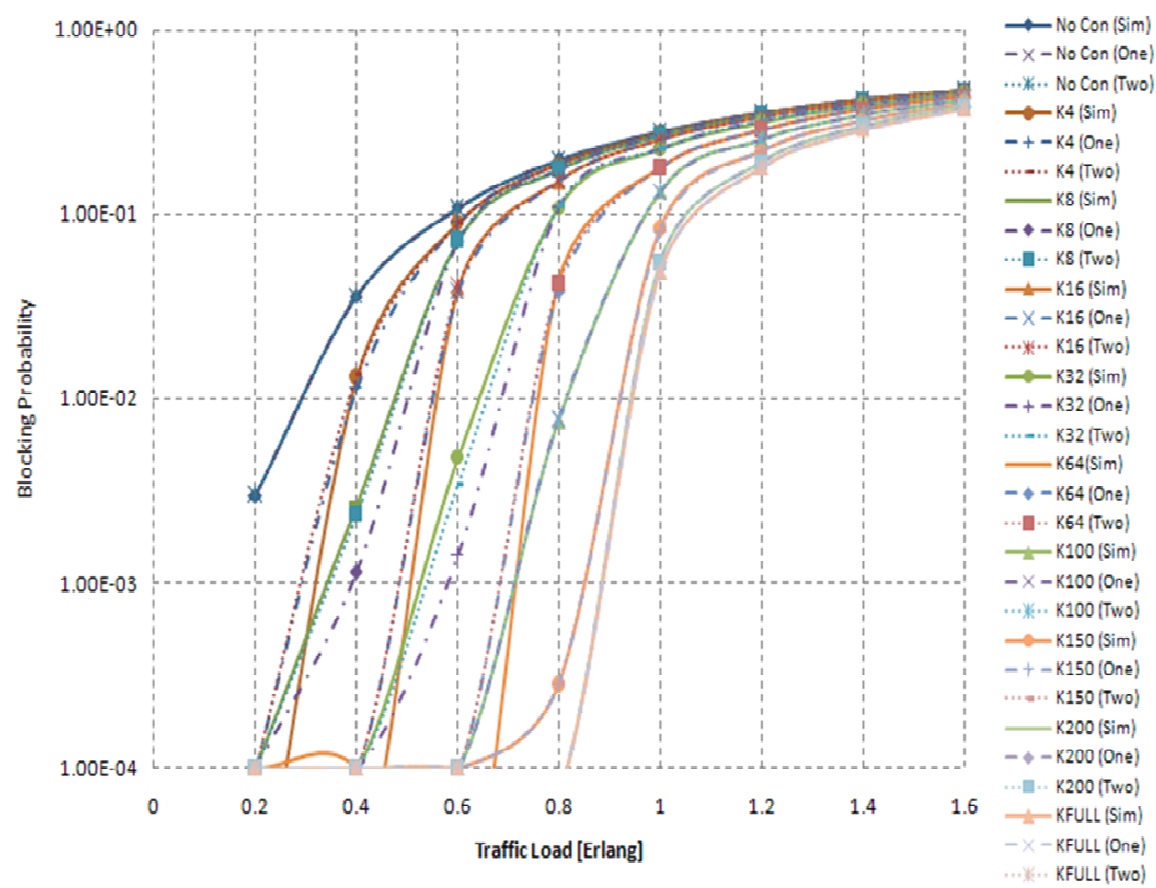

Fig. 4. Comparison of simulation and numerical results.

Fig. 4 shows the comparison of the results using numerical analysis and simulations. The results of simulation and two moment analysis agree well for small and large values of traffic, whereas there is a significant difference between the results of simulation and one moment analysis for the case of small value of traffic. For large value of traffic load simulation, one moment and two moment analysis result in well agreed model.

\section{Conclusion}

In this paper, we presented the analytical model for analyzing WDM switching networks with limited number of wavelength converters using overflow analysis method. Analytical methods using one moment and two moment analysis techniques of ERT method are presented and comparison of results is presented.

\section{References}

1. Glenstrup, A. J., Iverson, V. B.: Exact Evaluation of Blocking in WDM Networks, COM,Technical University of Denmark, (2001)

2. Yates, J. M., Rumsewicz, M. P., Lacey, J.P.R., Everitt, D.E.: Modeling Blocking Probabilities in WDM Networks with Fixed Alternate Routing, Australian Photonics Cooperative Research Centre, University of Melbourne, (1997) 
3. Subramanium, S., Somani, A. K., Azizoglu, M., Barry, R. A.: The Benefits of Wavelength Conversion in WDM Networks with Non-Poisson Traffic, IEEE Communication Letters, Vol. 3, No. 3, (1999)

4. Tripathi, T., Sivarajan, S. V.: Computing Approximate Blocking Probabilities in Wavelength Routed All Optical Networks with Limited Range Wavelength Conversion, IEEE Journal on Selected Areas in Communication, Vol 18, No 10, (2000).

5. Iverson, V. B.: Teletraffic Engineering Handbook- ITC in cooperation with ITU-D SG2, Geneva, (2003).

6. Jagerman, D. L.: Methods in Traffic Calculations, AT\&T Bell LaboratoriesTechnical Journal, Vol. 63, No. 7, (1984).

7. Rehman, A. A., Karim, A.: Modeling and Performance Analysis of WDM Switching Networks with a Limited Number of Wavelength Converters, IEEE-INMIC Conference, Karachi Pakistan, (2005). 